International Mathematical Forum, 2, 2007, no. 45, 2221 - 2230

\title{
Asymptotic Estimates of the Eigenvalues of Some Integral Operators
}

\author{
Yüksel Soykan \\ Zonguldak Karaelmas University \\ Faculty of Science and Art \\ Department of Mathematics \\ 67100, Zonguldak \\ Turkey
}

\begin{abstract}
In this paper, we give asymptotic estimates of the eigenvalues of certain positive Fredholm operators
\end{abstract}

Mathematics Subject Classification: 45C05, 45H05, 45P05, 30D55, 42B30

Keywords: Eigenvalues, Special Kernels, Positive Integral Operators, Hardy Spaces

\section{Introduction}

Throughout let $J$ be a fixed closed subinterval of the real line $\mathbb{R}$. Suppose that $D$ is a simply-connected domain containing the real closed interval $J$ and $\varphi$ is any function, which maps $D$ conformally onto $\Delta$, where $\Delta$ is the open unit disk of complex plain $\mathbb{C}$. Then let us define a function $K_{D}$ on $D \times D$ by

$$
K_{D}(\zeta, z)=\frac{\varphi^{\prime}(\zeta)^{\frac{1}{2}} \overline{\varphi^{\prime}(z)^{\frac{1}{2}}}}{1-\varphi(\zeta) \overline{\varphi(z)}} \quad \text { for all } \quad \zeta, z \in D
$$

for either of the branches of $\varphi^{\prime \frac{1}{2}}$. This function is independent of the choice of mapping function $\varphi$, see $[3$, p.410]. For example, if $D=\Delta$, then

$$
K_{D}(z, w)=\frac{1}{1-z \bar{w}} \quad(\text { take } \quad \varphi(z)=z)
$$


and if $D$ is the right half plane, then

$$
K_{D}(z, w)=\frac{1}{z+\bar{w}} \quad\left(\text { take } \quad \varphi(z)=\frac{z-1}{z+1}\right) .
$$

By restricting the function $K_{D}$ to the square $J \times J$ we obtain a compact symmetric operator $T_{D}$ on $L^{2}(J)$ defined by

$$
T_{D} f(s)=\int_{J} K_{D}(s, t) f(t) d t \quad\left(f \in L^{2}(J), s \in J\right) .
$$

This operator is always positive in the sense of operator theory (i.e. $(T f, f) \geq 0$ for all $\left.f \in L^{2}(J)\right)$, see [3]. We shall use $\lambda_{n}\left(T_{D}\right)$ to denote the eigenvalues of $T_{D}$.

The main aim of this work is to prove in detail the following theorem.

Theorem 1 If $D_{1}, D_{2}$ are two discs and if $D=D_{1} \cap D_{2}$ contains the real closed interval $J$, then

$$
\lambda_{n}\left(K_{D}\right) \simeq \lambda_{n}\left(K_{D_{1}}+K_{D_{2}}\right)
$$

where $a_{n} \simeq b_{n}$ means $a_{n}=O\left(b_{n}\right)$ and $b_{n}=O\left(a_{n}\right)$.

This is a special case of a theorem in [3, Theorem 1] but we give a different proof.

\section{Preliminaries And Factorization Through $E^{2}$}

To prove above Theorem 1 we need the following lemma but we recall some definitions first.

The space $H^{\infty}(\Delta)$ is just the set of all bounded analytic function on $\Delta$ with the uniform norm. For $1 \leq p<\infty, H^{p}(\Delta)$ is the set of all functions $f$ analytic on $\Delta$ such that

$$
\sup _{0<r<1} \frac{1}{2 \pi} \int_{0}^{2 \pi}\left|f\left(r e^{i \theta}\right)\right|^{p} d \theta<\infty .
$$

The $p$-th root of the left hand side of (1) here defines a complete norm on $H^{p}(\Delta)$. For more information on this spaces see [1, 2 and 4$]$. In the case of $p=2, H^{2}$ be the familiar Hardy space of all functions analytic on $\Delta$ with square-summable Maclaurin coefficients.

Let $D$ be a simply connected domain in $\overline{\mathbb{C}}=\mathbb{C} \cup \infty$ and let $\varphi$ be a Riemann mapping function for $D$, that is, a conformal map of $D$ onto $\Delta$. An analytic 
function $g$ on $D$ is said to be of (Smirnov) class $E^{2}(D)$ if there exists a function $f \in H^{2}(\Delta)$ such that

$$
g(z)=f(\varphi(z)) \varphi^{\prime}(z)^{\frac{1}{2}} \quad(z \in D)
$$

where $\varphi^{\prime \frac{1}{2}}$ is a branch of the square root of $\varphi^{\prime}$. We define $\|g\|_{E^{2}(D)}=\|f\|_{H^{2}(\Delta)}$. Thus, by construction, $E^{2}(D)$ is a Hilbert space with

$$
<g_{1}, g_{2}>_{E^{2}(D)}=<f_{1}, f_{2}>_{H^{2}(\Delta)}
$$

where $g_{i}(z)=f_{i}(\varphi(z)) \varphi^{\prime}(z)^{\frac{1}{2}}, \quad(i=1,2)$ and the map $U_{\varphi}: H^{2}(\Delta) \rightarrow E^{2}(D)$ given by

$$
U_{\varphi} f(z)=f(\varphi(z)) \varphi^{\prime}(z)^{\frac{1}{2}} \quad\left(f \in H^{2}(\Delta), z \in D\right)
$$

is an isometric bijection. For more information on this spaces see [ 1 and 6$]$. If $\partial D$ is a rectifiable Jordan curve then the same formula

$$
V_{\varphi} f(z)=f(\varphi(z)) \varphi^{\prime}(z)^{\frac{1}{2}} \quad\left(f \in L^{2}(\partial \Delta), z \in \partial D\right)
$$

defines an isometric bijection $V_{\varphi}$ of $L^{2}(\partial \Delta)$ onto $L^{2}(\partial D)$, the $L^{2}$ space of normalized arc length measure on $\partial D$ where $\partial D$ and $\partial \Delta$ denote the boundary of $D$ and $\Delta$ respectively. The inverse

$$
V_{\psi}=V_{\varphi}^{-1}: L^{2}(\partial D) \rightarrow L^{2}(\partial \Delta)
$$

of $V_{\varphi}$ is given by

$$
V_{\psi} g(w)=g(\psi(w)) \psi^{\prime}(w)^{\frac{1}{2}} \quad\left(g \in L^{2}(\partial D), w \in \partial \Delta, \psi=\varphi^{-1}\right) .
$$

Lemma 2 Suppose that $D$ is a disc or a codisc or a half-plane and $\gamma^{\prime} \subseteq \bar{D}$ be a circular arc (or a straight line) then for every $g \in E^{2}(D)$,

$$
\frac{1}{2 \pi} \int_{\gamma^{\prime}}|g(z)|^{2}|d z| \leq\|g\|_{E^{2}(D)}^{2}=\frac{1}{2 \pi} \int_{\partial D}|g(z)|^{2}|d z| .
$$

Proof. This is Corollary 1.3 to Lemma 1.2 in [5].

Suppose now that $D$ contains our fixed interval $J$. By restricting $\varphi$ to $J$ we obtain a linear operator $S_{D}: E^{2}(D) \rightarrow L^{2}(J)$ defined by

$$
S_{D} f(s)=f(s) \quad\left(f \in E^{2}(D), s \in J\right) .
$$

Then $S_{D}$ is compact operator and $T_{D}=S_{D} S_{D}^{*}$ is the compact, positive integral operator on $J$ with kernel $K_{D}$ :

$$
K_{D}(s, t)=\frac{\varphi^{\prime}(s)^{\frac{1}{2}} \overline{\varphi^{\prime}(t)^{\frac{1}{2}}}}{1-\varphi(s) \overline{\varphi(t)}}
$$

for all $s, t \in J$. This is proved in [3]. 
Definition 1 Let $H$ and $H^{\prime}$ be Hilbert spaces and suppose that $T$ is a compact, positive operator on $H$. If $S: H^{\prime} \rightarrow H$ is a compact operator such that $T=S S^{*}$, then $S$ is called a quasi square-root of $T$. We call $H^{\prime}$ the domain space of $S$.

Remark 1 Suppose that $D_{1}, D_{2}$ are simply-connected domains containing $J$ and let $T_{D_{1}}, T_{D_{2}}$ be continuous positive operators on a Hilbert space $L^{2}(J)$ and suppose that for each $i, S_{D_{i}}$ is a quasi square-root of $T_{D_{i}}$ with domain space $E^{2}\left(D_{i}\right)$. If $T_{+}=T\left(K_{D_{1}}+K_{D_{2}}\right)=T\left(\sum_{i=1}^{2} K_{D_{i}}\right)$ so that

$$
T_{+} f(s)=\int_{J}\left(K_{D_{1}}(s, t)+K_{D_{2}}(s, t)\right) f(t) d t \quad\left(f \in L^{2}(J), s \in J\right),
$$

then $T_{+}: L^{2}(J) \rightarrow L^{2}(J)$ is compact, positive integral operator and $T_{+}$has the quasi square-root $S_{+}: E^{2}\left(D_{1}\right)+E^{2}\left(D_{2}\right) \rightarrow L^{2}(J)$,

$$
S_{+}\left(f_{1}, f_{2}\right)=S_{D_{1}} f_{1}+S_{D_{2}} f_{2}
$$

so that

$$
\begin{aligned}
S_{+}\left(f_{1}, f_{2}\right)(s) & =S_{D_{1}} f_{1}(s)+S_{D_{2}} f_{2}(s) \\
& =f_{1}(s)+f_{2}(s) \quad\left(f \in L^{2}(J), s \in J\right) .
\end{aligned}
$$

Lemma 3 Let $T_{1}, T_{2}$ be compact operators on a Hilbert space $H$ and suppose that $S_{1}, S_{2}$ are quasi square-root of $T_{1}, T_{2}$ with domain $H_{1}, H_{2}$ respectively.

i) If there exists a continuous operator $V: H_{2} \rightarrow H_{1}$ such that $S_{2}=V S_{1}$ then $\left(T_{2} f, f\right) \leq k\left(T_{1} f, f\right)$ for some $k>0$ and so $\lambda_{n}\left(T_{2}\right) \simeq O\left(\lambda_{n}\left(T_{1}\right)\right) \quad(n \geq 0)$.

ii) If there exists continuous operators $V: H_{2} \rightarrow H_{1}$ and $W: H \rightarrow H$ such that $S_{2}=W S_{1} V$, then $\lambda_{n}\left(T_{2}\right) \simeq O\left(\lambda_{n}\left(T_{1}\right)\right)$.

Proof. See [3, page 407 ].

\section{Main Result}

Now we are ready to prove the theorem, which mentioned in the introduction. We prove that if $D_{1}, D_{2}$ are two discs containing the closed real interval $J$, then

i) $\quad \lambda_{n}\left(K_{D_{1} \cap D_{2}}\right)=O\left(\lambda_{n}\left(K_{D_{1}}+K_{D_{2}}\right)\right)$

ii) $\quad \lambda_{n}\left(K_{D_{1}}+K_{D_{2}}\right)=O\left(K_{D_{1} \cap D_{2}}\right)$.

What follows are the main steps in the proof of this theorem. Suppose that $D$ is a simply connected and bounded domain. Let $\varphi$ be a Riemann mapping function for $D$ and suppose that $\psi=\varphi^{-1}$ is the inverse function of $\varphi$. An analytic function $f$ on $D$ is said to be of class $H^{\infty}(D)$ if it is bounded on $D$. 
Proposition 4 If $\psi^{\prime} \in H^{1}$, then $H^{\infty}(D) \subseteq E^{2}(D)$.

Proof. Suppose that $f \in H^{\infty}(D)$. For $z \in D$, define $g(z)=f(\psi(z)) \psi^{\prime}(z)^{1 / 2}$. Then $f o \psi \in H^{\infty}(D)$ and $\psi^{\prime 1 / 2} \in H^{2}$. It follows that $($ fo $\psi) \psi^{\prime 1 / 2} \in H^{2}$. Hence $f \in E^{2}(D)$.

Proposition 5 Suppose that $\partial D$ is a rectifiable Jordan curve, then

i) $\psi^{\prime} \in H^{\prime}$

ii) Each function $f \in E^{2}(D)$ has a non-tangential limit $\tilde{f} \in L^{2}(\partial D)$. The map $f \rightarrow \widetilde{f}$ is an isometric isomorphism and $\|f\|_{E^{2}(D)}^{2}=\frac{1}{2 \pi} \int_{\partial D}|\widetilde{f}(z)|^{2}|d z|$.

iii) If $D$ is a Smirnov domain, then polynomials (thus $H^{\infty}$ ) are dense in $E^{2}(D)$.

iv) If $D$ is a convex region, it is a Smirnov domain.

$v) E^{2}(D)$ coincides with the $L^{2}(\partial D)$ closure of the polinomials if and only if $D$ is a Smirnov domain

Proof. See [1, pages 44, 170 and 173]. The definition of smirnov domain is given in [1, page 173]

Remark 2 If $f \in L^{2}(\partial \Delta)$, then the formula

$$
P_{0} f(z)=\frac{1}{2 \pi i} \int_{\partial \Delta} \frac{f(\zeta)}{\zeta-z} d \zeta=\frac{1}{2 \pi} \int_{\partial \Delta} f(\zeta) \frac{1}{1-\bar{\zeta} z}|d \zeta| \quad(|z|<1)
$$

defines a continuous linear operator $P_{0}: L^{2}(\partial \Delta) \rightarrow H^{2}(\Delta)=E^{2}(\Delta)$ and $\left\|P_{0}\right\|=1$. In fact, $P_{0}$ is essentially the orthogonal projection of $L^{2}(\partial \Delta)$ onto $H^{2}(\Delta)$. Note that if $f\left(e^{n i \theta}\right)=\sum_{n=-\infty}^{\infty} a_{n} e^{n i \theta}$ then $P_{0} f(z)=\sum_{n=0}^{\infty} a_{n} z^{n}$ $\left(a_{n} \in l^{2}(\mathbb{Z})\right)$.

Remark 3 There are similar results for any discs in [3, page 423]. That is, if $D$ is a disc, or codisc or half-plane, then the formula

$$
P f(z)=\frac{1}{2 \pi i} \int_{\partial D} \frac{f(\zeta)}{\zeta-z} d \zeta
$$

defines a continuous linear operator $P: L^{2}(\partial D) \rightarrow E^{2}(D)$ with $\|P\|=$ 1. Throughout suppose now that $D_{1}, D_{2}$ are plane discs and let $D=D_{1} \cap D_{2}$ contains the real closed interval J. For $k=1,2$, let $\gamma_{k}=\partial D_{k} \cap\left(D_{1} \cap D_{2}\right)$. We shall exhibit continuous operators

$$
N: E^{2}(D) \rightarrow E^{2}\left(D_{1}\right) \oplus E^{2}\left(D_{2}\right) \quad \text { and } \quad M: E^{2}\left(D_{1}\right) \oplus E^{2}\left(D_{2}\right) \rightarrow E^{2}(D) .
$$


To define $N$ suppose first that $G=\left\{f: f\right.$ is a polynomial in $\left.E^{2}(D)\right\}$. Note that then $\bar{G}=E^{2}(D)$ because $D$ is convex.

If $f \in G$, then for all $z \in D$, Cauchy's Integral Formula gives

$$
f(z)=\frac{1}{2 \pi i} \int_{\gamma_{1}} \frac{f(w)}{w-z} d w+\frac{1}{2 \pi i} \int_{\gamma_{2}} \frac{f(w)}{w-z} d w .
$$

For $f \in G$ and $1 \leq k \leq 2$, define a function $f_{k}$ on $D_{k}$ by

$$
f_{k}(z)=\frac{1}{2 \pi i} \int_{\gamma_{k}} \frac{f(w)}{w-z} d w \quad\left(z \in D_{k}\right)
$$

and define a function $\tilde{f}_{k}$ on $\partial D_{k}$ by

$$
\tilde{f}_{k}(z)=\left\{\begin{array}{ll}
f(\zeta) \quad \text { if } & \zeta \in \gamma_{k} \\
0 & \text { if } \quad \zeta \in \partial D_{k}-\gamma_{k}
\end{array} \quad\left(z \in \partial D_{k}\right)\right.
$$

Lemma 6 Suppose that $f \in G$ and $1 \leq k \leq 2$ then

i) $\tilde{f}_{k} \in L^{2}\left(\partial D_{k}\right)$ and $f_{k} \in E^{2}\left(D_{k}\right)$.

ii) The formula

$$
V_{1} f=\left(f_{1}, f_{2}\right)
$$

defines a continuous linear operator $V_{1}: G \rightarrow E^{2}\left(D_{1}\right) \oplus E^{2}\left(D_{2}\right)$ and so that $V_{1}$ has an extension $N$ by continuity to $E^{2}(D)$.

Proof i) Let $\varphi_{k}$ be a Riemann mapping function for $D_{k}$ and suppose $V_{\varphi_{k}}$ and $U_{\varphi_{k}}$ are as in Section 2.

Consider the Figure 1

where the map $P_{k}: L^{2}\left(\partial D_{k}\right) \rightarrow E^{2}\left(D_{k}\right)$ given by

$$
P_{k} f(z)=\frac{1}{2 \pi i} \int_{\partial D_{k}} \frac{f(\zeta)}{\zeta-z} d \zeta
$$

is a continuous linear operator with $\left\|P_{k}\right\|=1$ (from Remark 3 ).

Since $f_{k}=P_{k} \tilde{f}_{k}$, it follows that $f_{k} \in E^{2}\left(D_{k}\right)$. So $\left(f_{1}, f_{2}\right) \in E^{2}\left(D_{1}\right) \oplus$ $E^{2}\left(D_{2}\right)$. Since

$$
\begin{aligned}
\left\|f_{k}\right\|_{E^{2}\left(D_{k}\right)}^{2} & =\left\|P_{k} \tilde{f}_{k}\right\|_{E^{2}\left(D_{k}\right)}^{2} \leq\left\|\tilde{f}_{k}\right\|_{L^{2}\left(\partial D_{k}\right)}^{2}=\frac{1}{2 \pi} \int_{\gamma_{k}}|f(\zeta)|^{2}|d \zeta| \\
& \leq\|f\|_{E^{2}(D)}^{2}
\end{aligned}
$$




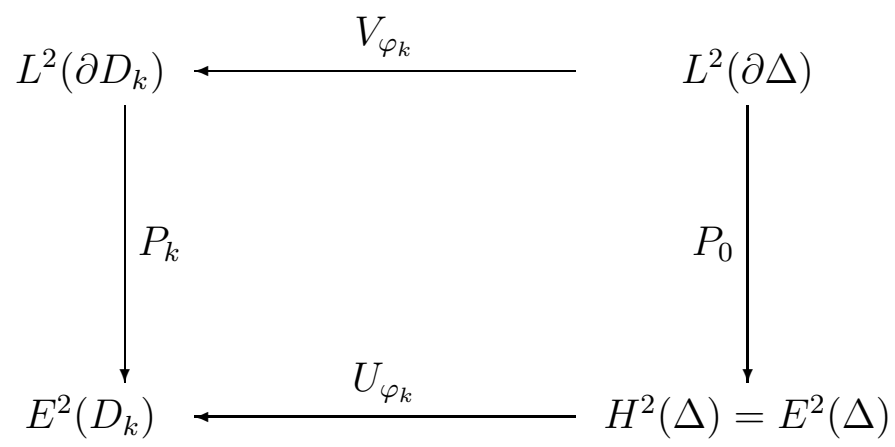

Figure 1:

and

$$
\begin{aligned}
\left\|V_{1} f\right\|_{E^{2}\left(D_{1}\right) \oplus E^{2}\left(D_{2}\right)}^{2} & =\left\|\left(f_{1}, f_{2}\right)\right\|_{E^{2}\left(D_{1}\right) \oplus E^{2}\left(D_{2}\right)}^{2}=\left\|f_{1}\right\|_{E^{2}\left(D_{1}\right)}^{2}+\left\|f_{2}\right\|_{E^{2}\left(D_{2}\right)}^{2} \\
& \leq 2\|f\|_{E^{2}(D)}^{2} .
\end{aligned}
$$

It follows that the map $f \rightarrow\left(f_{1}, f_{2}\right)$ is a continuous linear operator $G \rightarrow$ $E^{2}\left(D_{1}\right) \oplus E^{2}\left(D_{2}\right)$. Now suppose that $N$ is an extension by continuity to $E^{2}(D)$. Note that then $\|N\|^{2} \leq 2$.

If we denote $F=\bar{H}^{\infty}\left(D_{1}\right) \oplus H^{\infty}\left(D_{2}\right)$ then $\bar{F}=E^{2}\left(D_{1}\right) \oplus E^{2}\left(D_{2}\right)$.

Lemma 7 The map $V_{2}: F \rightarrow E^{2}(D)$, is given by

$$
V_{2}\left(f_{1}, f_{2}\right)(z)=f_{1}(z)+f_{2}(z), \quad\left(\left(f_{1}, f_{2}\right) \in F, \quad z \in D\right),
$$

is a continuous operator so that $V_{2}$ has an extension $M$ by continuity to $E^{2}\left(D_{1}\right) \oplus E^{2}\left(D_{2}\right)$.

Proof If $\left(f_{1}, f_{2}\right) \in F$ then by Propositions 4 and 5

$$
f_{i} \in H^{\infty}(D) \subseteq E^{2}(D) \quad(1 \leq i \leq 2)
$$

and

$$
V_{2}\left(f_{1}, f_{2}\right) \in H^{\infty}(D) \subseteq E^{2}(D) .
$$

So we have

$$
\begin{aligned}
\left\|V_{2}\left(f_{1}, f_{2}\right)\right\|_{E^{2}(D)}^{2}= & \left\|f_{1}+f_{2}\right\|_{E^{2}(D)}^{2} \\
= & \left\|f_{1}\right\|_{E^{2}(D)}^{2}+\left\|f_{2}\right\|_{E^{2}(D)}^{2}+2 \operatorname{Real}\left\langle f_{1}, f_{2}\right\rangle_{E^{2}(D)} \\
\leq & \left\|f_{1}\right\|_{E^{2}(D)}^{2}+\left\|f_{2}\right\|_{E^{2}(D)}^{2}+\left(\left\|f_{1}\right\|_{E^{2}(D)}^{2}+\left\|f_{2}\right\|_{E^{2}(D)}^{2}\right) \\
= & 2\left(\left\|f_{1}\right\|_{E^{2}(D)}^{2}+\left\|f_{2}\right\|_{E^{2}(D)}^{2}\right) \\
& (\text { by Corollary } 2) \\
\leq & 2\left(2\left\|f_{1}\right\|_{E^{2}\left(D_{1}\right)}^{2}+2\left\|f_{2}\right\|_{E^{2}\left(D_{2}\right)}^{2}\right) \\
= & 4\left\|\left(f_{1}, f_{2}\right)\right\|_{E^{2}\left(D_{1}\right) \oplus E^{2}\left(D_{2}\right)}^{2} .
\end{aligned}
$$


Hence $\left\|V_{2}\right\|^{2} \leq 4$ and $V_{2}$ is a continuous linear operator. Let now $M$ be extension by continuity to $E^{2}\left(D_{1}\right) \oplus E^{2}\left(D_{2}\right)$. Note that then $\|M\|^{2} \leq 4$.

\section{PROOF OF THEOREM 1}

i) Consider the Figure 2 where $V_{1}$ is as in Lemma 6

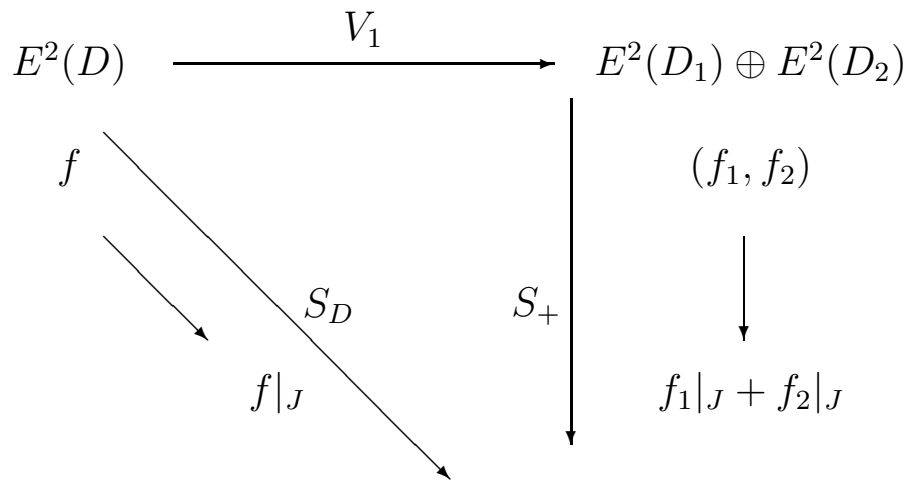

Figure $2 L^{2}(J)$

Note that here $T_{+}=S_{+} S_{+}^{*}$ and $T_{D}=S_{D} S_{D}^{*}$. By definition of $V_{1}$, we have $S_{D} f=S_{+} V_{1} f$ for every $f \in G$. Thus, by continuity of $V_{1}, S_{D} f=S_{+} N f$ for every $f \in E^{2}(D)$ and so $S_{D}=S_{+} N$.

So for $g \in L^{2}(J)$,

$$
\begin{aligned}
\left\langle S_{D} S_{D}^{*} g, g\right\rangle & =\left\|S_{D}^{*} g\right\|^{2}=\left\|N^{*} S_{+}^{*} g\right\|^{2} \\
& \leq\left\|N^{*}\right\|^{2}\left\|S_{+}^{*} g\right\|^{2}=\|N\|^{2}\left\langle S_{+} S_{+}^{*} g, g\right\rangle \\
& \leq 2\left\langle S_{+} S_{+}^{*} g, g\right\rangle
\end{aligned}
$$

That is,

$$
S_{D} S_{D}^{*} \leq 2 S_{+} S_{+}^{*}
$$

Hence by Lemma 3

$$
\lambda_{n}\left(S_{D} S_{D}^{*}\right) \leq 2 \lambda_{n}\left(S_{+} S_{+}^{*}\right)
$$

as required.

ii) Consider the Figure 3 where $V_{2}$ is as in Lemma 7 . By definition of $V_{2}$, it follows that $S_{+}\left(f_{1}, f_{2}\right)=S_{D} V_{2}\left(f_{1}, f_{2}\right)$ for every $\left(f_{1}, f_{2}\right) \in H^{\infty}\left(D_{1}\right) \oplus H^{\infty}\left(D_{2}\right)$. Thus, by continuity of $V_{2}$,

$$
S_{+}\left(f_{1}, f_{2}\right)=S_{D} M\left(f_{1}, f_{2}\right) \quad \text { for every } \quad\left(f_{1}, f_{2}\right) \in E^{2}\left(D_{1}\right) \oplus E^{2}\left(D_{2}\right)
$$




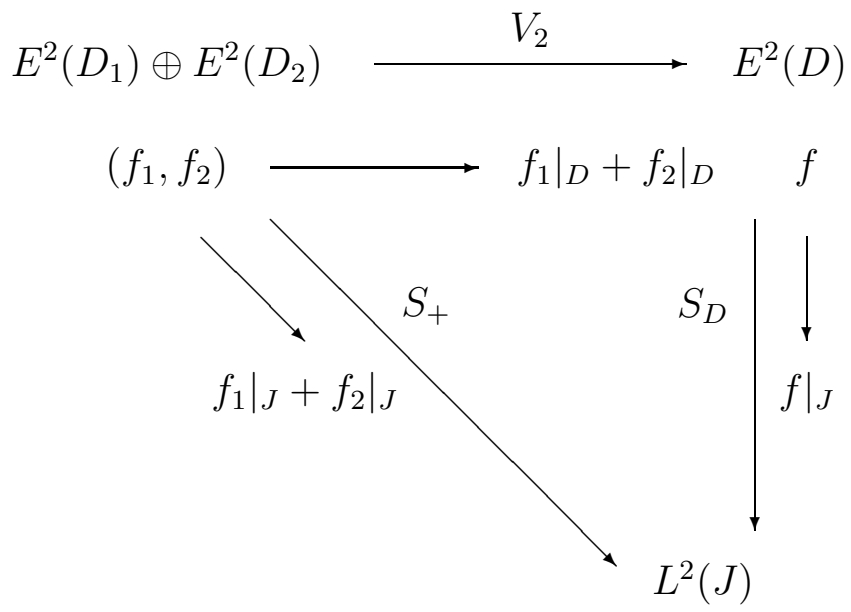

Figure 3:

and so $S_{+}=S_{D} M$. So for $g \in L^{2}(J)$, we have

$$
\begin{aligned}
\left\langle S_{+} S_{+}^{*} g, g\right\rangle & \leq\|M\|^{2}\left\langle S_{D} S_{D}^{*} g, g\right\rangle \\
& \leq 4\left\langle S_{D} S_{D}^{*} g, g\right\rangle
\end{aligned}
$$

i.e,

$$
S_{+} S_{+}^{*} \leq 4 S_{D} S_{D}^{*}
$$

Consequently, from Lemma 3,

$$
\lambda_{n}\left(S_{+} S_{+}^{*}\right) \leq 4 \lambda_{n}\left(S_{D} S_{D}^{*}\right)
$$

\section{References}

[1] Duren, P. L., Theory of Hp Spaces Academic Press, New York, 1970.

[2] Koosis, P., Introduction to Hp Spaces Cambridge University Press, Cambridge, Second Edition, 1998

[3] Little, G., Equivalences of positive integrals operators with rational kernels., Proc. London. Math. Soc. (3) 62 (1991), 403-426.

[4] Pommerenke, C., Boundary Behaviour of Conformal Maps, SpringerVerlag, Berlin, Heidelberg, 1992.

[5] Soykan Y., An Inequality of Fejer-Riesz Type, Cankaya University, Journal of Arts and Sciences, Issue: 5, May 2006, 51-60 
[6] Soykan, Y., On equivalent characterisation of elements of Hardy and Smirnov spaces., Int. Math. Forum. 2, 2007, no. 24, 1185 - 1191.

[7] Soykan, Y., Boundary behaviour of analytic curves, Int. Journal of Math. Analysis, Vol. 1, 2007, no. 3, 133 - 157.

Received: January 17, 2007 